\title{
MONITORING FLUCTUATIONS AT A SYNCHROTRON BEAM-LINE USING MATCHED ION CHAMBERS: 2. ISOLATION OF COMPONENT NOISE SOURCES, AND APPLICATION TO ATTENUATION MEASUREMENTS SHOWING INCREASED PRECISION BY TWO ORDERS OF MAGNITUDE
}

\author{
C. T. Chantler, C. Q. Tran, D. Paterson, D. J. Cookson†, Z. Barnea
}

School of Physics, University of Melbourne, Parkville, Victoria 3010, Australia

$†$ Australian Nuclear Science \& Technology Organisation, Private Mail Bag 1, Menai, NSW 2234

\& Chem-Mat-CARS-CAT (Sector 15, Bldg 434D), Argonne National Laboratory, 9700 S. Cass Avenue, Argonne, IL 60439

\section{ABSTRACT}

The significance of statistical fluctuations in a synchrotron beam is often neglected, with a consequent loss of precision or accuracy of up to two orders of magnitude. We illustrate this for the specific example of an X-ray attenuation measurement. Since all X-ray measurements involve either scattering or absorption (or both), the net potential gain in precision is similar for all such experiments, including crystallographic and XAFS determinations. We demonstrate the net gain with data obtained with two matched ion chambers on a monochromatised bending magnet beam at the Photon Factory, Tsukuba. Isolating and measuring component contributions to the overall fluctuations allows a robust determination of the limiting experimental precision. This approach also determines the absolute incident flux without measuring the absolute photon count. The type of statistical analysis described is not only a post-facto diagnostic tool but, by being incorporated into the experiment on-line, can provide a real-time optimising intervention in the measurement process.

Keywords: determination of statistic, synchrotron source, fluctuations, ion chambers, $0.01 \%$ attenuation measurement 


\section{Introduction}

The companion paper ${ }^{1}$ has discussed a model of the experimental geometry involving paired ion chambers as monitor and detector upstream and downstream with a suitable scattering or attenuating sample interposed. It explored assumptions that synchrotron sources are distributed in a symmetric Gaussian manner, and revealed problems in basing analysis on quoted specifications for a beam-line, or in relying upon operator-provided beam current profiles for precision experiments. The problem raised is accentuated by the presence of significant additional normally-distributed and non-Gaussian fluctuations.

Use of the model, optimised experimental conditions, and a correct understanding of correlated signals allowed apparent experimental limitations at the $1 \%$ - $7 \%$ uncertainty level to be reduced to $0.01 \%$ $0.03 \%$. This is a major improvement which can make otherwise impractical experiments possible.

In the specific field of absorption coefficient measurement, this increase in precision allows fundamental atomic physics and existing theory to be critically investigated, ${ }^{2}$ and allows improvement upon earlier work by up to two orders of magnitude. We cite here issues regarding the physics of scattering contributions, ${ }^{3}$ the relativistic correction factor in the atomic form factor, ${ }^{4}$ investigations of XAFS and other structure near absorption edges, ${ }^{5}$ and discrepancies between theoretical predictions of absorption coefficients. ${ }^{6}$ As with any area of research, an order of magnitude improvement should allow the resolution of many outstanding questions.

This paper develops the a priori model ${ }^{1}$ into an on-line diagnostic tool by isolating the noise contributions, analysing their correlated statistical signatures, and utilising them to indicate corrective measures needed to provide statistically optimised information. It also discusses criteria for collecting such optimised data.

\section{2: The four independent measures of noise provided by the data and their significance}

The experimental geometry (upstream monitor and downstream detector) gives four independent measurable parameters: the noise (observed standard deviation) in the upstream ion chamber $\sigma_{\text {obsup }}$; the noise in the downstream ion chamber $\sigma_{\text {obsdown }}$; the correlation coefficient between the two chamber readings $R_{\text {updown }}$; and the standard deviation $\sigma_{\text {obsratio }}$ of the point-to-point ratio.

These all measure some combination of independent fluctuations, but with different weights. Thus, accurate compilation of all four measures of association allows access to information on different combinations of noise components. This is shown explicitly by the equations below, where reference is 
made to relative s.d's as identified and observed in Tables 1-4, below.

$$
\begin{aligned}
& {\sigma_{\text {obsup }}}^{2}=\sum_{\mathrm{i} \text { component }}{\sigma_{i, \text { rel }}}^{2} \approx \sigma_{\text {decay }}^{2}+\sigma_{\text {time }}^{2}+\sigma_{\text {zeroup }}^{2}+\sigma_{\text {up }}^{2}+\sigma_{\text {absup }}^{2}+\sigma_{\text {ionup }}^{2}
\end{aligned}
$$

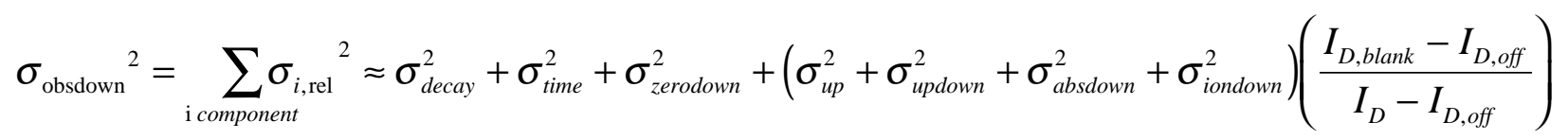

The meaning of each component is given in the first column of Table 4, below. Further explanation of the symbols is given explicitly elsewhere. ${ }^{1}$ While most of the symbols are obvious or defined in Table 4, some remarks are needed for the details of implementation of these formulae.

Each equation has two parts. The first is a simple statistical identity and the second approximation is its implementation in our model. The exact equations apply in a model-independent manner if all correlated contributions are exactly known. The approximations given in the same equations represent our assumptions both as to the type of component contributions and as to the full correlation matrix. In other words, if we have identified the time-dependence, dominant correlations and dominant contributions to fluctuations across all time-scales and measurements, then the approximate relations $[\approx]$ should be close to identities and the model should agree well with all the experimental data in each independent set.

We differentiate between the flux of photons after monchromation $I_{\text {up }}$, the fluxes of photons absorbed per second in the upstream monitor $I_{\text {absup }}$ or downstream detector $I_{\text {absdown }}$, and the integrated current reading recorded by the detectors $I_{\mathrm{M}}$ and $I_{\mathrm{D}}$. The percentage standard deviation (or coefficient of variation) contributions from zero offset noise $\left(\sigma_{\text {zeroup }}\right.$ and $\left.\sigma_{\text {zerodown }}\right)$, are computed from their absolute standard deviations, divided by the actual detector or monitor current reading with the beam on, $I_{\mathrm{M}}$ or $I_{\mathrm{D}}$ respectively. The attenuation factor involving $I_{\mathrm{D}}$ (current reading) is computed from experimental values, and requires a measurement of the straight-through beam (i.e. no attenuator $I_{\mathrm{D} \text {,blank }}$ ) for each attenuated measurement. Electronic bias offsets lead to signals $I_{\mathrm{M}, \text { off }}$ and $I_{\mathrm{D}, \text { off }}$ in the absence of any photon flux.

The remaining two independent observables are the correlation coefficient between the two chamber readings $R_{\text {updown }}$; and the standard deviation $\sigma_{\text {obsratio }}$ of the point-to-point ratio:

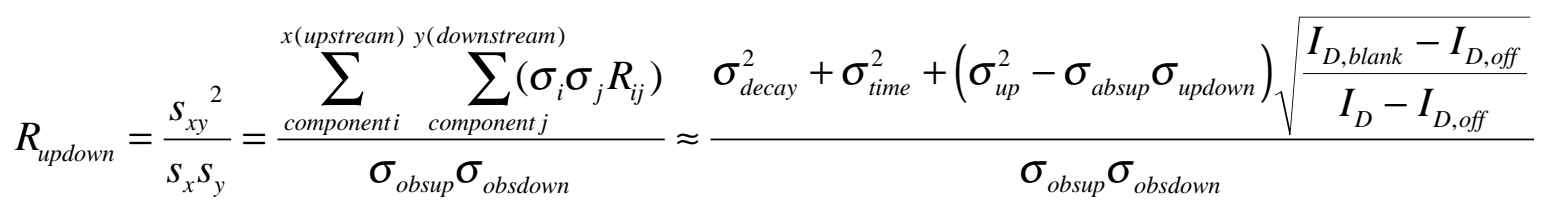




$$
\begin{aligned}
& \left(\frac{\sigma_{\text {obsratio }}}{\left(I_{D}-I_{D, \text { off }}\right) /\left(I_{M}-I_{M, \text { off }}\right)}\right)^{2}=\left(\frac{\sigma_{\left(\left(I_{D}-I_{D, \text { off }}\right) /\left(I_{M}-I_{M, \text { off }}\right)\right)}}{\left(I_{D}-I_{D, \text { off }}\right) /\left(I_{M}-I_{M, \text { off }}\right)}\right)^{2} \\
& =\sigma_{\text {obsup }}^{2}+\sigma_{\text {obsdown }}^{2}-2 R_{\text {updown }} \sigma_{\text {obsup }} \sigma_{\text {obsdown }} \\
& \left(\frac{\sigma_{\text {obsratio }}}{\left(I_{D}-I_{D, \text { off }}\right) /\left(I_{M}-I_{M, \text { off }}\right)}\right)^{2} \approx \sigma_{\text {zeroup }}^{2}+\sigma_{\text {zerodown }}^{2}+\sigma_{\text {ionup }}^{2} \\
& +\left(\sigma_{\text {iondown }}^{2}+\sigma_{\text {absdown }}^{2}\right)\left(\frac{I_{D, \text { blank }}-I_{D, \text { off }}}{I_{D}-I_{D, \text { off }}}\right)+\left(\sigma_{\text {absup }}+\sigma_{\text {updown }} \sqrt{\frac{I_{D, \text { blank }}-I_{D, \text { off }}}{I_{D}-I_{D, \text { off }}}}\right)^{2}
\end{aligned}
$$

The approximations in Eqs (3) and (4) are very good when no 'accidental' correlations occur between causally uncorrelated components. Accidental correlation can occur if a very thick attenuator is placed between the detectors when the uncorrelated downstream noise dominates and the signal is also very weak. Under these conditions an accidental correlation between this 'uncorrelated' component and some other contributing fluctuation may become significant and lead to a correlation coefficient of \pm 0.5 or so. This would imply that conditions and samples are not correctly chosen for the experiment.

Equation (4a) may be implemented using the approximate relations given in Eqs (1) - (3), which is not identical to the approximate relation given by Eq. (4b), but both represent analysis of the same dominant correlations. Hence a check of the validity of the model is to compare the results of Eq. (4a) with those of Eq. (4b) and with directly observed data.

Because of the large variability of the decay trend, ${ }^{1}$ a short-term and a long-term estimate were generally compared to determine the apparent standard deviation of the pooled variance due to the trend, and hence the actual standard deviation underlying the trend:

$$
\begin{aligned}
& \sigma_{\text {decay }}=\left\{\begin{array}{l}
\sigma_{\text {observedshort-termtrend }} \\
\sigma_{\text {observedlong-termitrend }}
\end{array}\right\} \times \sqrt{\frac{\sum_{i=1}^{N} i^{2}-\left(\frac{N+1}{2}\right)^{2}}{N-1}}, \mathrm{~N} \text { observations (points per scan) if trend not removed(5a) } \\
& \sigma_{\text {decay }}=\left\{\begin{array}{l}
\sigma_{\text {observedshort-termitrend }} \\
\sigma_{\text {observedlong-termtrend }}
\end{array}\right\} \times 1, \text { if trend removed }
\end{aligned}
$$

The long-term trend is the relative change in flux per point measurement (i.e. 3 seconds, 20 seconds or whatever) determined over the full duration of an independent set of scans. The short-term trend is the relative change in flux for the same duration but determined from a single scan (11 or 21 
points). If the trend is uniform these measures will be consistent with the long-term measure more accurate; however, if the beam is unstable over the duration of acquisition of the set of data, the long-term trend is meaningless and the short-term trend is much more reliable. The results of both Eq. (5a) and (5b) are consistent, underscoring their validity; and Eq. (5a) is the obvious derived variance consequential on determining the mean of any series with a linear trend.

\section{3: What independent noise components can be directly investigated?}

For the observed $\sigma_{\text {obsup }}$ (Eq. (1)), the upstream noise signal weights each source of variance equally. For the downstream detector fluctuation $\sigma_{\text {obsdown }}$, components are weighted differently - the attenuating sample increases the relative statistical noise components with the decrease in flux, while the other contributions to downstream noise are similar to upstream monitor values. The zero offset noise can dominate in the downstream variance for strongly attenuating targets. The correlation coefficient $R_{\text {updown }}$ gives the ratio of the correlated noise contributions (positive $\sigma_{\text {time }}, \sigma_{\text {decay }}, \sigma_{\text {up }}$ minus negative $\sigma_{\text {absup }} \sigma_{\text {updown }}$ ) compared to the total. The point-to-point ratio s.d. $\sigma_{\text {obsratio }}$ omits positively correlated contributions, being composed only of uncorrelated and anti-correlated components. The balance between these contributions changes significantly as the X-ray energy varies, from $5 \mathrm{keV}$ to $20 \mathrm{keV}$, allowing exploration of particular components at different energies.

Hence the timing error, the decay trend, short-term discontinuities in flux, the negatively correlated components, the positively correlated components and the uncorrelated components may all be separately investigated. A suitable model-based assumption must finally be invoked, but this can be tested against the data. Errors in the model, presented above and developed below, could involve omitted noise components, inadequate description of the correlation matrix, incorrect scalings with time, overestimated components or incorrect relative magnitudes of components. However, the consistency of the model predictions for an enormous range of results, where absolute s.d's have been explicitly varied through factors of over 100 , strongly confirms most details of this discussion at the level claimed.

Component percentage s.d's are simply given by photon or charge-counting statistics:

$$
\begin{aligned}
& \sigma_{u p}=\frac{1}{\sqrt{I_{u p} t}} \\
& \sigma_{\text {absup }}=\frac{1}{\sqrt{I_{a b s u p} t}}=\frac{1}{\sqrt{I_{u p} A_{i o n} t}}
\end{aligned}
$$


Here $A_{\text {ion }}$ is the fraction of photons absorbed in the active region of the ion chamber, so that $I_{u p} A_{i o n} t$ is simply the total number of photons absorbed in the active region of the upstream monitor.

$$
\sigma_{\text {ionup }}=\frac{1}{\sqrt{I_{\text {up }} A_{\text {ion }} t\left(E_{k e V} / 2 E_{\text {ionpair }}\right)}}
$$

The incident $\mathrm{X}$-ray energy is $\mathrm{E}_{\mathrm{keV}}$, and the energy to produce an ion pair in the ion chamber is $\mathrm{E}_{\text {ionpair }}$, so that $E_{k e V} / 2 E_{\text {ionpair }}$ is just the number of charges per photon absorbed.

$$
\begin{gathered}
\sigma_{\text {absdown }}=\frac{1}{\sqrt{I_{\text {absdown }} t}}=\frac{1}{\sqrt{I_{u p} A_{\text {ion }}\left(1-A_{\text {air }}\right) t\left(1-A_{\text {ion }}\right)}} \\
\sigma_{\text {iondown }}=\frac{1}{\sqrt{I_{\text {up }} A_{\text {ion }} t\left(1-A_{\text {air }}\right)\left(1-A_{\text {ion }}\right)\left(E_{\text {keV }} / 2 E_{\text {ionpair }}\right)}} \\
\sigma_{\text {updown }}=\sqrt{\frac{A_{\text {ion }}}{I_{\text {up }} t\left(1-A_{\text {ion }}\right)}}
\end{gathered}
$$

\section{4: Measured photon and other noise contributions to observed standard deviations}

Determined incident flux, and associated uncertainties in flux and in observed noise are given in Table 1. The detailed measurements conducted are summarised in Table 2, giving the different timescales, energies and tests of reproducibility investigated. Table 3 explicitly summarises the large range of relative s.d's in the upstream monitor, the downstream detector and the point-to-point ratio observed in independent scans, together with the consequent variation in the observed correlation coefficient $R$. Our model must provide good agreement with observed results for correlation coefficients varying across almost the full possible range. The model (and observed results) include a scaling with time. This method can determine (in the first row of Table 1) the incident flux to $\sigma(\mathrm{I}) / \mathrm{I}=20 \%$, for fluxes varying by factors of 40 and for widely varying incident X-ray energies. This determination of incident flux relates to our observed upstream measurements; since the method is also successful for the downstream observations, the accuracy should be valid for much larger ranges of flux.

Downstream observations probed fluxes reduced by some $3 \times 10^{-5}$, with the flux determination becoming poorer but still useful at this low-flux limit. We achieve this without actually measuring the flux, but only by measuring the noise distribution. The confidence of our result is based on the consistency of 
consecutive flux determinations, and on the 10\%-40\% agreement of observed/predicted ratios of all measures $\left(\sigma_{\text {obsup }}, \sigma_{\text {obsdown }} \& \sigma_{\text {obsratio }}\right)$ for 5-8 independent data scans simultaneously, in each data set (Table 1). Each scan involves widely differing attenuation and probes the scaling of noise over a long baseline (Table 3). All predictions must agree with the observed \%s.d's for the defined photon fluxes, simultaneously. Each set of data scans probes $\sigma_{\text {obsup }}$ covering a range over a factor of four or twelve, which is a good probe of the noise components. $\sigma_{\text {obsratio }}$ is probed simultaneously over a factor of 30 . The range of $\sigma_{\text {obsdown }}$ probed in any set of data scans reaches a factor of 100 , or a range of downstream fluxes of $10^{4}$. Predicted results agree with observed results on all these temporal and flux scales.

Individual noise contributions for the investigation are presented in Table 4. These results depend in a simple manner upon the photon flux incident on the detector. Most dependences estimated prior to the experiment, based on reported beam and detector characteristics, are accurate. As presented in Table 2 of the companion paper, ${ }^{1}$ reported values in Table 4 are based on one second measurement times, and the observed scaling with other durations is indicated in the last column. Values of $\sigma_{\text {updown }}$ and $\sigma_{\text {absdown }}$ in Table 4 are based on 'blank' measurements (with no sample or attenuator) - all attenuated measurements will have increased variances as discussed.

Two results are presented in the rows for $\sigma_{\text {zeroup }}$ and $\sigma_{\text {zerodown }}$. The 'blank' or no attenuator limit represents the beam attenuated only by the air gap and the upstream detector, while the no beam limit represents the beam fully attenuated, thereby leaving the residual zero offset noise. Noise in these rows is effectively a simple counting limit - the uncertainty corresponding to this particular noise contribution is well represented by \pm 1 count.

Our a priori estimates of noise component magnitudes (Ref. 1) were usually accurate. Estimates of time dependences for the timing error and zero offset were inadequate, though they may be accurate for time-scales below one second. The number of photons estimated from the bending magnet computed output, collimation and monochromator efficiency, is accurate within factors of $3(5 \mathrm{keV}), 4(10 \mathrm{keV})$ and 6 (20 keV) (Table 1 versus predictions in companion paper). There is an intrinsic factor of two uncertainty in the predictions of Ref. 1 (based on optimal performance of the beam), as the beam may be at its maximum or minimum current before filling. The timing estimate was accurate within a factor of two. In poorly optimised settings, the actual amplifier noise was much larger. The decay estimate was in agreement with expectations but varied much more dramatically as a function of time than expected. The zero offset noise 
and discontinuities in beam current were also more significant than predicted.

The simple estimates ${ }^{1}$ of component relative s.d's were generally accurate within a factor of two. Derived results for component relative s.d's are much more accurate, with uncertainties estimated as 10\% $20 \%$ in any given series of data. Hence our approach is very reliable for both understanding and optimising data collected at synchrotrons.

\section{5: Can we optimise the statistic on-line to the precision required, as we collect data?}

It is probably obvious by now that the answer to this question is yes. We certainly achieved this. The value of a method for improving the limiting precision of results by an order of magnitude cannot be overestimated. In the final analysis, it is also worth the effort simply to be able to define contributing errors. The limitation in any given experiment will of course still depend upon detectors, collimation, divergence and all other parameters of the experiment.

Additionally, the on-line determination of photon and other noise allows a rapid estimation of the limitations of a particular beam-line, or of a particular day of synchrotron operation, which might invalidate a given type of experiment. The detailed approach could be quite time-consuming. However, by using a system of equations and fitting programs as discussed here, the precise consequences of gas pressure, amplifier range, detector tuning etc. can be quantified within the first hour of data collection. Since some modifications decrease error contributions of particular components by orders of magnitude, this is a very effective use of beam-time.

\section{6: How to optimise the precision}

The optimum precision involves correct analysis, allowing for all correlation between noise contributions. The overall correlation coefficient should be positive and close to unity.

The ranges of $R_{\text {updown }}$ listed in Table 3 include specific data sets where the correlation was negative. In general this can be due to many characteristics of the experimental chain. In our cases this was sometimes due to a very heavy attenuator which allowed zero offset noise to dominate, and sometimes this was due to spurious short-term trends in detectors (dominated by accidental correlations between formally uncorrelated noise contributions). Hence longer data series would have minimised this, and use of appropriate target thicknesses would have resolved other cases. 
To demonstrate that this is a resolvable issue we present Figure 1, in which we plot the correlation coefficient $R_{\text {updown }}$ as a function of energy. Clearly the correlation is optimised, and almost all results are highly correlated as intended. Further, for comparisons between monitor and detector with no attenuator interposed, $R_{\text {updown }}$ is always above 0.9 , and often around 0.99 . The figure shows a larger spread at the very low and very high energies. This is due to the explicit use of a range of thicknesses and the wide variation of attenuation over the energy range investigated. The thickest attenuator demonstrated the problem of accidental correlation, while the thinner attenuators were largely unaffected. There is also a trend for the value of $R_{\text {updown }}$ to decrease at higher energies, which is due to the detector efficiency declining with energy. This could be further optimised by changing the detector gas.

The net improvement over poorly optimised investigations is quite variable. Perhaps the best comparison is that given in section 11 of the companion paper, regarding the precision of relative thickness determinations. The precision of attenuation point-to-point ratios may be one or two orders of magnitude better than s.d's using other methods. Accuracy may also be dramatically improved. Absolute intensities are needed in many experiments, but even the determination of ratios of intensities is dramatically improved by this approach.

For two particular data series discussed in Ref. 1, the point-to-point ratio s.d's were $0.15 \%$ and $0.045 \%$ respectively. The standard error of the ratio is a factor of 3-5 smaller in these scans, approaching $0.03 \%$ to $0.01 \%$. These optimised precisions compare to estimates using pooled variances of $1 \%$ to $7 \%$ precision, which have been limiting precisions in many previous literature results in the attenuation field. This relative improvement is also represented by the use of inappropriate equations (Eqs (1) or (7) in Ref. 1), as opposed to optimised equations with proper inclusion of correlation (Eqs (6), (8) or (9) therein).

A much more extensive study along these lines is presented in Figure 2. We explicitly collected two independent sets of results at different times with no sample or attenuator for an extended series of measurements. This reflects the consistency of results achieved using optimised matched detectors but with poor analysis, not incorporating the effects of correlation between signals. The result plotted in Figure 2 mimics the use of Eq. (7) of the companion paper. This figure shows the observed variance using monitor and detector ratios with offsets, but with random pairing of data (i.e. with no correlation).

These results have a precision of $0.1 \%$ to $0.2 \%$, compared to the pooled variance of $1 \%-3 \%$ based on thickness variation of samples. If the measurements were not normalised at all, we would get a 
distribution reflected in Figure 2 of Ref. 1, dominated by the decay contribution and with an enhanced relative standard error, of some few percent. These should not be limitations in a well-designed experiment.

Figure 3 shows the consistency based on correct normalisation of the same data. The consistency is one or two orders of magnitude improved compared with Figure 2 or with results neglecting the monitor signal (Figure 2, Ref. 1). This consistency is represented in the derived \%s.d.'s after inclusion of correlation. The consistency of the data does indeed lie at the $0.03 \%$ to $0.01 \%$ level. The importance of a monitor is clear, but this is not sufficient unless correlated analysis is performed on the data, in which case the predicted improvement of up to two orders of magnitude in precision is indeed observed.

One series of measurements remains consistently higher than the other (Figure 3). Interestingly, the statistical precision is still not the limiting factor in these results. Even at this level, understanding the small systematic discrepancy may in future work lead to a limiting precision below the $0.01 \%$ level.

\section{Conclusions}

Introducing a monitor counter in an experiment permits a dramatic increase in the accuracy of results. However, ignoring the correlation between the monitor and detector in such experiments leads to large and unnecessary imprecision. Failure to optimise experimental and detection conditions also leads to large systematic errors and imprecision.

Optimisation of all contributions to the observed noise, and appropriate analysis with incorporation of all correlation, can permit a precision up to two orders of magnitude better than that achievable by previous, alternate approaches. Optimisation includes detector gas selection, optimisation of amplifier settings, selection of the optimal experimental attenuation and optimising for strong positive correlation.

Many details regarding individual components of the statistic of a detected signal on a synchrotron line may be determined to high accuracy, without absolute calibration of the detector efficiency. We have determined the absolute photon count incident in an experiment to within $\sigma(\mathrm{I}) / \mathrm{I}=20 \%$, without counting the photons. The accuracy of observed standard deviations $\sigma_{\text {obsup }}, \sigma_{\text {obsdown }}$ and $\sigma_{\text {obsratio }}$ is limited by sample size, flux and the observed attenuation, but is typically $\sigma_{\text {obsup }}=10 \%$ (and similarly for the other three observed measures). The component contributions to relative standard deviations $\left(\sigma_{\text {up }}, \sigma_{\text {ebeam }}, \sigma_{\text {absup }} ; \sigma_{\text {updown }}\right.$, $\sigma_{\text {absdown }}, \sigma_{\text {ionup }}, \sigma_{\text {iondown }}, \sigma_{\text {time }}, \sigma_{\text {decay }}, \sigma_{\text {zeroup }}, \sigma_{\text {zerodown }}$ and $\sigma_{\text {amp }}$ ) have all been determined from model predictions, observed standard deviations and the correlation coefficient $R_{\text {updown }}$. Each measurement investigated regimes where different noise components were significant, so that the accuracy of individual component standard deviations (e.g. $\sigma_{\text {up }}$ ) varied from $10 \%$ to $50 \%$.

Fairly simple models account for the observations and can be used for on-line diagnostics and optimisation of the statistic. In many experiments, the zero offset noise and the amplifier noise can limit experimental precision beyond acceptable limits unless conditions are optimised. 


\section{Acknowledgements}

We would like to acknowledge R.J. Garrett and D.C. Creagh for their helpful discussions. This work was performed at the Australian National Beamline Facility with support from the Australian Synchrotron Research Program, which is funded by the Commonwealth of Australia under the Major National Research Facilities program.

\section{References}

1. Chantler, C.T., C. Q. Tran, D. Paterson, Z. Barnea, D. J. Cookson, X-ray Spectrometry (this issue)

2. Chantler, C.T., Barnea, Z., Tran, C., Tiller, J., Paterson, D. (1999), “Precision X-ray optics for fundamental interactions in atomic physics, resolving discrepancies in the X-ray regime,” Optical \& Quantum Electronics 31, 495-505.

3. Chantler, C.T., Barnea, Z. (1999) "Resolution of a discrepancy of X-ray attenuation measurements of silicon in the energy range 25-50 keV," J. Phys. Condensed Matter 11 (1999) 4087-4091.

4. Creagh, D.C. and McAuley, W. (1995), 'X-ray dispersion corrections,' section 4.2.6, pp206-222, in "International Tables for Crystallography, Vol. C,” A.J.C. Wilson, Ed. (Kluwer Academic).

5. Chantler, C.T. (2000) J. Phys. Chem. Ref. Data (in press).

6. Chantler, C.T. (1995) J. Phys. Chem. Ref. Data 24, 71-643. 